\title{
Rapid mixing chemical oxidative polymerization: an easy route to prepare PANI coated small-diameter CNTs/PANI nanofibres composite thin film
}

\author{
G VENKATA RAMANA ${ }^{a}$, BALAJI PADYA ${ }^{\mathrm{b}}$, VADALI V S S SRIKANTH ${ }^{\mathrm{a} * *}$ and P K JAIN \\ ${ }^{a}$ School of Engineering Sciences and Technology (SEST), University of Hyderabad, Hyderabad 500 046, India \\ ${ }^{\mathrm{b}}$ Centre for Carbon Materials, International Advanced Research Centre for Powder Metallurgy and New Materials \\ (ARCI), Hyderabad 500 005, India
}

MS received 15 January 2013; revised 9 March 2013

\begin{abstract}
Composite thin film containing polyaniline (PANI) coated small diameter carbon nanotubes (SDCNTs)/PANI nanofibres (NFs) has been prepared using an easy in situ rapid mixing chemical oxidative polymerization method. SDCNTs thin film was obtained using thermal chemical vapour deposition method in a separate experiment, whilst PANI NFs are formed in situ during the synthesis of composite. In the composite, PANI coated SDCNTs are uniformly distributed among PANI NFs. The presence of SDCNTs during the composite synthesis does not influence the nucleation and growth of PANI NFs. Raman analysis shows a good interaction between PANI and SDCNTs. Room temperature d.c. electrical sheet resistance of SDCNTs/PANI NFs composite thin film surface is three orders lesser than that of PANI NFs thin film (PANI NFs have the same morphology as in the composite) synthesized using the same method but without the presence of SDCNTs.
\end{abstract}

Keywords. Thin films; surface structure; nano-structures; CNTs; PANI.

\section{Introduction}

Combining 1D nanostructures like carbon nanotubes (Ajayan and Zhou 2001) and polyaniline nanofibres (PANI NFs) (Huang and Kaner 2004) to form a composite is expected not only to produce attractive advanced functional materials (Saini et al 2009; Ding et al 2011; Yan et al 2011), but also to stimulate pluridisciplinary research activities. PANI matrix composites can be obtained by using in situ polymerization of aniline in carbon nanomaterial suspensions (Gedela et al 2012); here, the problems of poor solvability and/or dispersion of the constituent materials in the solvents used in synthesis protocols can be overcome. In this study, an easy method (in situ chemical oxidative polymerization) to synthesize small diameter carbon nanotubes (SDCNTs) and PANI NFs containing composite thin film surface will be demonstrated.

\section{Experimental}

SDCNTs thin film was grown using ET3000 (Firstnano, USA) thermal chemical vapour deposition system. Iron (catalyst)-coated silicon wafer was used as the substrate.

*Author for correspondence (vvsssse@uohyd.ernet.in)
After placing the substrate in the furnace of the deposition system, the furnace was evacuated and then purged with Ar gas. Subsequently, $\mathrm{CH}_{4}(2 \mathrm{SLM})$ was cracked at $900{ }^{\circ} \mathrm{C}$ in the presence of hydrogen gas $(0.2 \mathrm{SLM})$ for $10 \mathrm{~min}$. The furnace was purged with Ar gas at a flow rate of 1 and 5 SLM during heating and cooling, respectively.

In the next step, $250 \mathrm{~mL}$ of aqueous $\mathrm{HCl}$ was taken in a $1 \mathrm{~L}$ round-bottom flask and $20 \mathrm{~mL}$ of vacuum-distilled aniline was added to it and subsequently thin film containing SDCNTs was placed at the bottom of the flask. The reaction mixture was then ultrasonicated for $1 \mathrm{~h}$. Next, the reaction mixture was cooled to $0{ }^{\circ} \mathrm{C}$ using an ice bath. The polymerization was then initiated by rapidly adding ammonium perdisulfate (APS) to the reaction mixture $(\mathrm{HCl}+$ aniline + SDCNTs thin film). During APS addition, temperature of the reaction mixture was maintained in the range of $0-5{ }^{\circ} \mathrm{C}$. After APS addition, the reaction mixture was left stirring for $4 \mathrm{~h}$. The resultant thin film was then removed from the flask and was washed with water, then with methanol, and finally with diethyl ether. The thin film was then dried at $90{ }^{\circ} \mathrm{C}$ under vacuum for $48 \mathrm{~h}$. For comparison purposes, thin film constituted only by PANI NFs was also synthesized following the above-mentioned protocol except for the addition of SDCNTs thin film to the reaction mixture.

Field emission scanning electron microscope (FESEM) (Model Zeiss Ultra 55) and transmission electron micro- 
scope (TEM) (Model FEI Technai $\mathrm{G}^{2} \mathrm{~S}$-Twin) were used to study the morphology of the synthesized materials; they were operated at accelerating voltages of 5 and $200 \mathrm{kV}$, respectively. Micro Raman spectroscopic study was carried out using LabRam HR800 Raman spectrometer; $514.5 \mathrm{~nm}$ green line of $\mathrm{Ar}^{+}$ion laser was used as the excitation source. Four-probe arrangement was used to measure d.c. electrical sheet resistance of the thin film surface. Keithley 6221 and 2182A were used as the current source and voltmeter, respectively.

\section{Results and discussion}

Secondary- and transmission-electron micrographs of only SDCNTs and PANI NFs constituting thin film surfaces are shown in figure 1 . It can be observed from the micrographs (figures $1(\mathrm{a}$ and $\mathrm{b})$ ) that SDCNTs have high aspect ratios (diameter $\sim 10-25 \mathrm{~nm}$ and length few $\mathrm{mm}$ ). It can also be observed that SDCNTs are randomly oriented and in most of the areas, they are agglomerated. On the other hand, PANI NFs (figures 1 (c and d)) are $\sim 20$ $30 \mathrm{~nm}$ in diameter and $\sim$ sub-microns in length. Figure 2 shows secondary electron surface morphology of SDCNTs and PANI NFs containing thin film. It can be clearly observed from the micrographs that the surfaces of SDCNTs are uniformly coated with PANI. The diameter of PANI-coated SDCNTs has a very narrow range with an average of $\sim 250 \mathrm{~nm}$. It can also be observed that the length of the coated SDCNTs is the same as the original length of SDCNTs. Interestingly, PANI NFs in the composite thin film maintained similar morphology and diameter range $(\sim 20-30 \mathrm{~nm})$ as in the case of only PANI
NFs containing thin film (figures 1(c and d)). These observations indicate that the addition of SDCNTs thin film in the reaction mixture has not influenced the formation of PANI NFs. Another observation is that PANI coated SDCNTs are randomly oriented in the PANI NFs matrix. Such pockets of morphology are observed uniformly on the entire surface of the composite thin film. Figure 2(b) shows the formation of core (SDCNT) and shell (PANI) in the composite thin film. It should also be noted that owing to the large length of SDCNTs, there can be a discontinuity in the coverage of SDCNTs with PANI as shown in figure 2(b); however, the observations of such discontinuities are found to be only few in number. These discontinuities could be plausibly avoided by choosing SDCNTs thin film constituted by SDCNTs possessing slightly lower aspect ratio when compared to the SDCNTs under present discussion.

Raman spectra obtained from the samples are shown in figure 3. As for the SDCNTs (figure 3(a)), the characteristic Raman bands have been observed (Dresselhaus et al 2010). The bands at $\sim 1595$ (G band) and $\sim 1350 \mathrm{~cm}^{-1}$ (D band) indicate graphitic nature and disorderness pertaining to SDCNTs, respectively. It is clearly observed in the electron micrographs that SDCNTs maintained high aspect ratio resulting in negative curvature type defects on the surfaces which in turn have resulted in the appearance of the D band. A weak band at $\sim 298 \mathrm{~cm}^{-1}$ is attributed to the radial breathing mode (RBM) related to the diameter of single-walled carbon nanotubes (SWCNTs). As for the thin film surface containing PANI NFs, and SDCNTs/PANI NFs, characteristic stretching Raman modes ( $\mathrm{Yu}$ et al 2005) corresponding to bonds in PANI

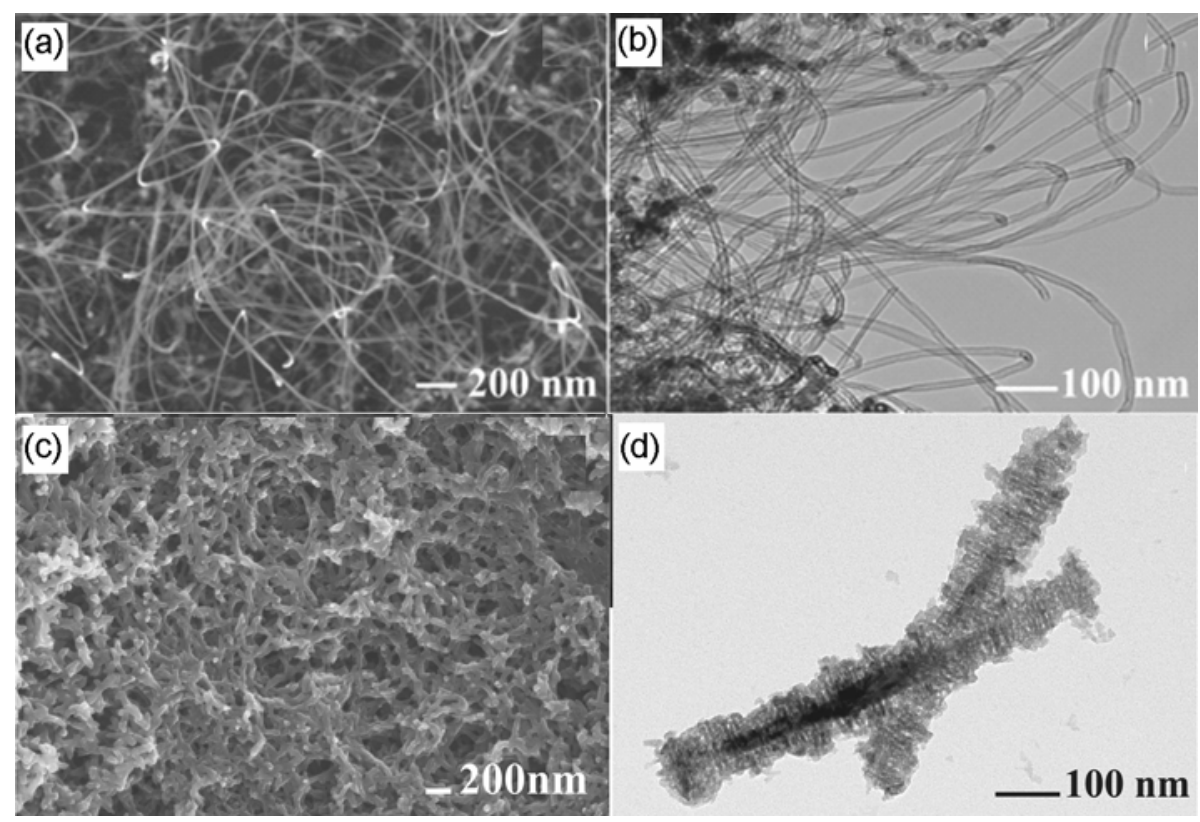

Figure 1. Secondary electron plane view images of (a) SDCNTs and (c) PANI NFs and transmission electron plane view images of (b) SDCNTs and (d) PANI NFs. 


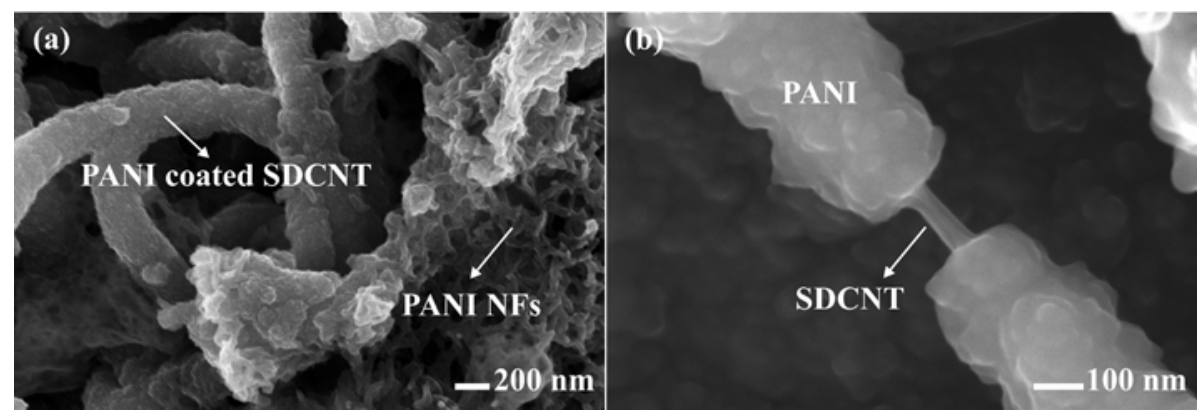

Figure 2. Secondary electron plane view images of (a) composite thin film surface containing PANI coated SDCNTs and PANI NFs and (b) core (SDCNT)/shell (PANI) structure.

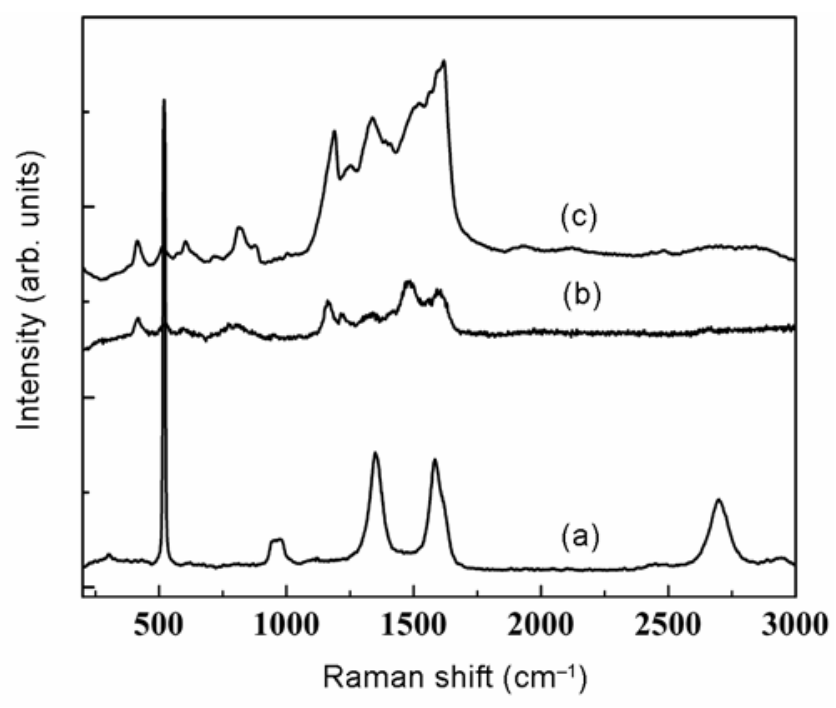

Figure 3. Raman spectra obtained from (a) SDCNTs, (b) PANI NFs and (c) composite (PANI coated SDCNTs and PANI NFs constituting) thin film surfaces.

Table 1. Raman shift values (with corresponding bonds in PANI) obtained from samples.

\begin{tabular}{ll}
\hline Raman shift $\left(\sim \mathrm{cm}^{-1}\right)$ & \multicolumn{1}{c}{ Assignment } \\
\hline 423 & C-N-C torsion \\
752 & C-N-C bending \\
1170 & C-H bending \\
1328 & C=N stretching \\
1483 & C-C + C-N stretching \\
1589 & C-C (Qui) stretching \\
\hline
\end{tabular}

are identified (table 1). However, in the case of SDCNTs/ PANI NFs composite thin film, there is a considerable red shift in PANI characteristic bands corresponding to $\mathrm{C}=\mathrm{N}$ and $\mathrm{C}-\mathrm{C}$ stretching modes. This shift indicates (Cochet et al 2001) that there is an interaction between PANI and SDCNTs in the composite. Moreover, the disappearance of the characteristic $\mathrm{G}$ band pertaining to SDCNTs from the Raman spectrum corresponding to the composite thin film surface indicates that the SDCNTs are coated with PANI.

The formation mechanism of PANI NFs and SDCNTs/ PANI NFs composite thin film surface is interpreted on the similar lines of a previously reported proposal (Liang et al 2002). In the present synthesis protocol, aniline was at first mixed in aq. $\mathrm{HCl}$ solution and subsequently, SDCNTs thin film was placed in that mixture and subjected to stirring. During this process, some of the aniline molecules can plausibly get adsorbed on the surfaces of the SDCNTs; this can be due to $\pi-\pi$ interaction between hexagonally bonded graphitic layers of SDCNTs and $\mathrm{C}=\mathrm{N}$ (qunioniod), which is in the backbone of aniline monomer. This type of interaction is evident from Raman analysis (red shift in $\mathrm{C}=\mathrm{N}$ Raman band corresponding to PANI). Remaining aniline molecules get dispersed in the aq. $\mathrm{HCl}$ suspension. At the moment of rapid mixing of APS in the reaction mixture (now, aq. $\mathrm{HCl}+$ aniline + SDCNTs), aniline is ready to get polymerized into PANI. But there are two nucleation sites, bulk solution and surfaces of SDCNTs. Due to the dilution levels used in the reaction, PANI NFs have formed both in the bulk solution and on all other surfaces except on the surfaces of SDCNTs. However, heterogeneous nucleation and growth of PANI have taken place on the surfaces of SDCNTs resulting in the core/shell type structures.

D.C. electrical sheet resistance of the composite thin film constituted by SDCNTs and PANI NFs has been found to be $\sim 605 \Omega$, which is almost three orders less when compared to the value $(\sim 16.4 \mathrm{k} \Omega)$ measured in the case of the thin film constituted by only PANI NFs. This shows that SDCNTs' presence has an obvious influence on the electrical conductivity of the composite thin film. Effective site-selective interaction between $\pi$-bonds in the aromatic ring of PANI and graphitic structure of carbon nanostructures was found to be the reason that strongly assists charge-transfer reaction between the two components causing such enhancement in electrical conductivity. The Raman analysis clearly showed that there is an obvious interaction between SDCNTs and PANI. However, the interaction has to be further explored. 


\section{Conclusions}

Composite thin film surface containing SDCNTs and PANI NFs has been synthesized using an easy in situ rapid mixing chemical oxidative polymerization method. Using this method, the complication of carbon nanostructures' dispersion in aniline solution can be avoided while preparing composites. In the composite thin film surface prepared in this work, PANI coated SDCNTs are uniformly distributed among PANI NFs. Raman analysis shows a good interaction between PANI and SDCNTs. Room temperature D.C. electrical sheet resistance of SDCNTs/PANI NFs composite thin film surface is three orders lesser than that of PANI NFs thin film.

\section{Acknowledgements}

The authors thank Prof. D Narayana Rao and Dr S Srinath, School of Physics (University of Hyderabad), for generously allowing us to use Raman spectrometer and FESEM, respectively. The authors also thank Ms. Deepthi, School of Physics (University of Hyderabad), for helping us with Raman measurements and Mr M Durga Prasad, Centre for Nanotechnology, University of Hyderabad, for helping us with TEM. (GVR) extends his gratitude to CSIR for its financial support through a Senior Research
Fellowship. (VVSSS) thanks UGC, India, for its financial support through a major research scheme vide no. F. 41993/2012 (SR).

\section{References}

Ajayan P M and Zhou O Z 2001Carbon nanotubes: synthesis, structure, properties and applications (eds) M S Dresselhaus, G Dresselhaus and Phaedon Avouris (Heidelberg: SpringerVerlag) p 406

Cochet M, Maser W K, Benito A M, Callejas M A, Martínez M T, Benoit J M, Schreiber J and Chauvet O 2001 Chem. Commun. 161450

Ding M N, Tang Y F, Gou P P, Reber M J and Star A 2011 Adv. Mater. 23536

Dresselhaus M S, Jorio A, Hofmann M, Dresselhaus G and Saito R 2010 Nano Lett. 10751

Gedela R V, Padya B, Srikanth V V S S, Jain P K, Padmanabham G and Sundararajan G 2011 Carbon 495239

Huang J and Kaner R B 2004 J. Am. Chem. Soc. 126851

Liang L, Liu J, Windisch C F, Exarhos G J and Lin Y 2002 Angew. Chem. Int. Ed. 413665

Saini P, Choudhary V, Singh B P, Mathur R B and Dhawan S K 2009 Mater. Chem. Phys. 113919

Yan X B, Tai Z X, Chen J T and Xue Q J 2011 Nanoscale 3 212

Yu Y J, Che B, Si Z H, Li L, Chen W and Xue G 2005 Synth. Met. 150271 